\title{
Qualidade dos cuidados de enfermagem em terapia intensiva: avaliação por meio de auditoria operacional
}

\author{
Quality of nursing care in intensive therapy: evaluation through operational auditing
}

Calidad de los cuidados de enfermería en terapia intensiva: evaluación por medio de auditoría operacional

\author{
Elaine Fátima Padilha', Laura Misue Matsuda" \\ ' Hospital Universitário do Oeste do Paraná. Cascavel-PR, Brasil. Universidade Estadual de Maringá, \\ Departamento de Enfermagem, Programa de Mestrado em Enfermagem. (Mestranda) Maringá-PR, Brasil. \\ " Universidade Estadual de Maringá, Departamento de Enfermagem, \\ Programa de Mestrado em Enfermagem. Maringá-PR, Brasil.
}

Submissão: 7/1/2010 Revisão: 21/1/2011 Aprovação: 11/8/2011

\begin{abstract}
RESUMO
Este estudo objetivou avaliar a qualidade do cuidado de enfermagem de uma Unidade de Terapia Intensiva para Adultos. Os dados de cinquenta clientes com mais de três dias de internação foram coletados por meio da observação, do exame físico e da análise do prontuário do paciente, utilizando-se um Roteiro de Auditoria Operacional. Os cuidados considerados de qualidade foram os que obtiveram percentual de respostas positivas $\geq 70 \%$, como aqueles relacionados à Segurança Física $(71 \%)$ e Utilização de Equipamentos ( $72 \%)$. A Atividade Física ( $28 \%$ ) e Oxigenação/Ventilação $(29 \%)$ foram os cuidados com percentuais mais baixos de respostas positivas. Conclui-se que há urgência em se executar ações de educação continuada no serviço investigado, porque a maioria dos itens e subitens de cuidados não foi considerada de qualidade.
\end{abstract}

Descritores: Auditoria de enfermagem; Qualidade da assistência à saúde; Avaliação em enfermagem; Cuidados intensivos; Unidade de Terapia Intensiva.

\section{ABSTRACT}

This study aimed to evaluate the quality of nursing care of an Adult Intensive Care Unit. Data from fifty in-hospital patients, who stayed in ICU for more than three days, were collected through observation, physical examination and patients' medicalinformation records analysis, using an Operational Auditing Plan. Care considered of quality were those that obtained positive answer percentage $\geq 70 \%$, as those related to Physical Safety $(71 \%)$ and Equipment Use $(72 \%)$. Physical Activity $(28 \%)$ and Oxygenation/Ventilation (29\%) reached the lowest scores. We concluded that is urgent to implement continuing education actions in the service studied, since most care items and sub-items did not present the quality needed.

Key words: Nursing audit; Quality of health care; Nursing assessment; Intensive care; Intensive Care Unit.

\section{RESUMEN}

Este estudio tuvo como reto evaluar la calidad del cuidado de enfermería de una Unidad de Terapia Intensiva Adulto (UTI-A). Los datos de 50 clientes con más de tres días de internación fueron colectados a través de la observación, del examen físico y del análisis del prontuario del paciente, utilizándose un Guión de Auditoría Operacional. Los cuidados considerados de calidad fueron los que obtuvieron porcentaje de respuestas positivas $\geq 70 \%$, como aquellos relacionados a la Seguridad Física (71\%) y Utilización de Equipamientos (72\%). La Actividad Física $(28 \%)$ y Oxigenación/Ventilación $(29 \%)$ fueron los cuidados con porcentajes más bajos. Se ha concluido que hay urgencia en ejecutarse acciones de educación continuada en el servicio investigado, porque la mayoría de los ítems y sub-ítems de cuidados no fue considerada de calidad.

Palabras clave: Auditoria de enfermería, Cualidad de la asistencia de salude; Evaluación en enfermería; Cuidados intensivos; Unidades de terapia intensiva. 


\section{INTRODUÇÃO}

Por meio da literatura, é possível constatar que, na área da saúde, existe uma grande busca pela qualidade e isso tem impetrado propostas e mudanças significativas no Sistema Único de Saúde (SUS) e nos modelos de gestão das instituições. As mudanças em prol da qualidade também podem ser notadas na postura dos profissionais de saúde, os quais apresentam preocupação crescente com a melhoria contínua, visando a excelência dos serviços ${ }^{(1)}$.

Apesar de se notar que a postura da valorização da qualidade já se faz presente em algumas instituições hospitalares do país e também entre os profissionais da área, observa-se a necessidade de maior envolvimento e compromisso por parte dos sujeitos, no sentido de que a qualidade nos serviços por eles prestados seja efetiva ${ }^{(2)}$.

Além do envolvimento dos profissionais e das instituições, a qualidade dos produtos/serviços requer o conhecimento da realidade e dos resultados de cada serviço oferecido à população para que se conheçam os pontos positivos e negativos que possibilitam promover reflexões e ações voltadas à melhoria dessa qualidade. Nessa perspectiva, as informações que atuam na melhoria dos processos, devem ser obtidas por meio de métodos sistemáticos, denominados de avaliação da qualidade.

A avaliação da qualidade, no modo como é referido neste estudo, consiste em uma ferramenta proposta pelo sistema Gestão da Qualidade (GQ), o qual surgiu no âmbito industrial, em meados do Século XX e que nos últimos anos tem se difundido no setor da saúde ${ }^{(3)}$.

A GQ compreende a busca da qualidade por meio da melhoria contínua dos processos das instituições, com foco principal no trabalho em equipe e na constante verificação e solução dos problemas, por meio da prevenção/eliminação dos erros ou inconformidades ${ }^{(4)}$.

Na Enfermagem, observa-se que alguns princípios do sistema da GQ têm sido empregados com bons resultados, como é o caso da avaliação dos cuidados por meio da Auditoria da Qualidade, que consiste num processo sistemático de avaliação, baseado nas anotações de enfermagem constantes no prontuário do paciente/cliente e na observação direta das suas condições, no próprio ambiente onde o cuidado é realizado ${ }^{(5-6)}$.

O processo de avaliação dos cuidados de enfermagem por meio da Auditoria da Qualidade pode ser realizado de forma periódica ou continuamente. Na periódica, estabelece-se um período de tempo para a realização da auditagem e, na contínua, a auditagem é realizada de forma permanente, no cotidiano de trabalho ${ }^{(6-7)}$. Quanto à natureza de sua realização, a auditoria pode ser normal ou específica, e, no tocante à limitação, pode ser total ou parcial ${ }^{(8)}$.

No que se refere à forma de intervenção, a Auditoria pode ser considerada interna ou externa. A primeira possibilita uma avaliação mais acurada, pois o processo de auditagem é feito por uma pessoa da própria instituição, beneficiada pelo fato de o auditor conhecer a estrutura administrativa, a cultura organizacional, as tecnologias e as metas do serviço avaliado.
A auditoria externa é realizada por pessoas de fora da instituição, que não possuem dependência administrativa para com ela, nem envolvimento afetivo com os indivíduos, e isso pode ser mais eficaz, porque permite recomendações finais mais críticas e criteriosas acerca dos resultados observados ${ }^{(7-8)}$.

Com relação ao tipo, a Auditoria da Qualidade pode ser retrospectiva ou operacional. A retrospectiva, realizada por meio de revisão sistemática, compreende a análise de dados provindos do prontuário do paciente, após sua alta da instituição/unidade. Já a operacional, se relaciona à verificação de como se processa o cuidado "in loco", por meio da observação direta, da entrevista informa/formal e, também, da análise de documentos ${ }^{(5)}$.

No Brasil existem diversos estudos sobre a aplicação da Auditoria da Qualidade do tipo retrospectiva, em instituições hospitalares. Porém, em se tratando da auditoria operacional, observa-se que as publicações referentes a este tema nas principais Bases de Dados disponibilizadas online - LILACS (Literatura Latino Americana e do Caribe em Ciências da Saúde), MEDLINE (Literatura Internacional em Ciências da Saúde), BDENF (Base de Dados de Enfermagem) e SciELO (Scientifc Eletronic Library Online) - são ainda escassas.

No âmbito da terapia intensiva, local onde as mínimas faIhas podem gerar graves consequências aos pacientes e à equipe, a adoção de uma prática avaliativa realizada por meio da Auditoria Operacional é de suma importância porque, ao ser realizada in loco, as falhas podem ser corrigidas ou evitadas "no tempo". Condutas assim tendem a favorecer o paciente que recebe um cuidado de melhor qualidade; e também a equipe de enfermagem, que evita implicações de ordem ética e legal ${ }^{(9)}$.

Considerando os benefícios que a Auditoria da Qualidade pode proporcionar à melhoria do gerenciamento do cuidado de enfermagem, questiona-se: $\mathrm{O}$ processo de avaliação, fundamentado na Auditoria Operacional, possibilita identificar a qualidade dos cuidados de enfermagem realizados em uma Unidade de Terapia Intensiva Adulto (UTI-A) de um hospital universitário público? Para responder a essa questão se propõe a realização deste estudo que tem como objetivo avaliar por meio da Auditoria Operacional, a qualidade do cuidado de enfermagem de uma UTI-A.

Justifica-se a realização deste estudo porque, na Enfermagem, investigações acerca das inconformidades/falhas detectadas por meio da Auditoria Operacional em terapia intensiva são escassas e, além disso, o conhecimento produzido poderá fomentar futuras pesquisas e ações de melhorias no Serviço.

\section{METODOLOGIA}

Estudo do tipo descritivo, observacional e de análise documental, com abordagem quantitativa, realizada no período de 1 de maio a 31 de julho de 2009, com 50 pacientes que permaneceram mais de três dias (72 horas) internados em uma UTI-A de um hospital de ensino público da região oeste do Paraná.

O instrumento de coleta de dados consistiu em um formulário no qual constava o Roteiro para Auditoria Operacional elaborado pela Assessoria de Controle de Qualidade da 
Assistência de Enfermagem (ACQAE) do Hospital Universitário de Londrina-PR, e descrito por $\operatorname{Haddad}^{(7)}$, composto por oito itens e 65 subitens:

- Item I - Higiene e Conforto, com oito subitens: cuidados com a boca; ordem dos cabelos; realização de tricotomia facial; aparamento das unhas dos pés e mãos; ausência de irritações e obstruções em sondas e drenos; correto posicionamento do paciente na cama; presença de curativos limpos e secos; e ordem/limpeza do leito do paciente.

- Item II - Atividades Físicas, com cinco subitens: programação de mudança de decúbito em paciente dependente; formação de Úlcera por Pressão (UP) nas últimas 24 horas; colocação adequada da tração ortopédica; colocação correta de tábuas para os pés, sacos de areia, travesseiros ou suportes; e cuidados corretos com gessos e talas.

- Item III - Segurança Física, composto por doze subitens: cuidados de enfermagem quanto à identificação correta e completa do paciente; acomodação segura na cadeira de rodas ou poltrona; posicionamento adequado das grades da cama; ausência de queda do leito nas últimas 24 horas; limpeza e adequado posicionamento das restrições de membros; presença de campainha ao alcance do paciente; segurança e adequação do mobiliário do boxe; adequação do equipamento elétrico do boxe; presença de objetos limpos acumulados no boxe; presença de objetos contaminados acumulados no boxe; limpeza do ambiente; e agendamento de exames.

- Item IV - Nutrição e Hidratação, com quinze subitens: troca dos equipos de microgotas a cada 72 horas; presença de data e instalação dos equipos de PVC a cada 72 horas; troca dos equipos de bomba de infusão a cada 72 horas; ausência de sangue coagulado na parte interna do equipo de soro/medicações; ausência de sangue coagulado na parte externa do equipo de soro/medicações; identificação correta dos equipos de soro/medicações; identificação completa e correta dos soros/medicações instalados; identificação das punções venosas periféricas realizadas; troca das punções venosas periféricas a cada 72 horas; presença de permeabilidade nos cateteres externos; ausência de soroma em membros superiores; troca dos equipos de dieta a cada 24 horas; limpeza dos equipos de dieta; presença de equipos coloridos para dieta e posicionamento do frasco da dieta acima da cabeça do paciente.

- Item V - Oxigenação e Ventilação, com cinco subitens: proteção e manutenção adequada de inaladores; instalação e troca adequada dos nebulizadores; ausência de secreção em traqueostomia; limpeza do curativo de traqueostomia; e instalação correta e com qualidade do oxigênio.

- Item VI - Eliminações, com sete subitens: presença de curativo ou coletor adequado em dreno de penrose; adequado enchimento e posicionamento de ostomias e bolsas coletoras; posicionamento adequado e manutenção de pressão negativa em dreno de porto-vac; instalação adequada do dreno de tórax; identificação adequada do dreno de tórax; correto posicionamento da sonda nasogástrica/nasoenteral; e adequada fixação do cateter vesical de demora.

- Item VII - Utilização de Equipamentos, com apenas um subitem referente à utilização da forma correta dos equipamentos.

- Item VIII - Unidade de Terapia Intensiva, composto por doze subitens: presença de prescrição de enfermagem para todos os pacientes; presença mínima de duas evoluções diárias; registro da realização de um exame físico por período; registro de sinais vitais (pressão arterial, frequência cardíaca, frequência respiratória, oximetria, glicemia capilar, pressão venosa central) e balanço hídrico de duas em duas horas; presença de camas com cabeceiras removíveis e grades laterais; presença de sistema fechado de coletor de urina; presença de roupa em número suficiente para a demanda; presença de cuidados preventivos para UP; ausência de obstrução, irritação local e infecção em drenos, coletores, sondas, cateteres e coletores; realização de orientação familiar; presença de controle de ruído no setor; e realização de antissepsia nas mãos, antes e após procedimentos no setor.

Para todos os subitens da segunda parte do formulário (Roteiro de Auditoria Operacional) existiam três possibilidades de respostas, sim, não e não se aplica. Para cada subitem, somente uma possibilidade de resposta era permitida.

A coleta dos dados ocorreu nos primeiros 30 minutos do plantão seguinte ao plantão em que os cuidados de enfermagem de rotina eram executados. A realização da Auditoria Operacional teve duração média de 30 minutos por paciente e se pautou na observação das condições materiais e dos equipamentos utilizados pelo paciente; na realização de exame físico e, quando necessário, consulta ao seu prontuário.

Os dados oriundos do formulário preenchido foram categorizados e compilados em bancos de dados separados e informatizados no programa Microsoft Office Excel ${ }^{\circledR} 2007$. A seguir, as planilhas provenientes destes bancos foram importadas para o programa Statistical Analisys System 9.1.3 (SAS) para a realização da análise estatística.

Para a determinação da qualidade dos cuidados avaliados por meio do Roteiro de Auditoria Operacional foi realizado o cálculo da Freqüência Relativa das respostas positivas obtidas para todos os itens e subitens do roteiro, a fim de identificar o Índice de Positividade (IP).

A partir do cálculo do IP foi realizada a classificação dos itens e subitens com base em Carter apud Saupe e Horr (10) descrito por Haddad ${ }^{(7)}$ que classificam o cuidado de acordo com o IP obtido, sendo: IP de 100\% - cuidado Desejável; IP entre 90 e 99\% - cuidado Adequado; IP entre 80 e $89 \%$ - cuidado Seguro; IP entre 70 a 79\% - cuidado Limítrofe e IP abaixo de $70 \%$ - cuidado Sofrível. Para fins deste estudo foram considerados de qualidade somente os itens do Roteiro de Auditoria Operacional que apresentaram IP igual ou superior a $70 \%(\mathrm{IP} \geq 70 \%)$.

A apresentação dos dados será realizada por meio de uma tabela contendo o IP e a Classificação de Qualidade para cada item do Roteiro. 
No que concerne aos aspectos éticos, para fazer parte do estudo foi solicitada a assinatura do Termo de Consentimento Livre e Esclarecido do participante (cliente/paciente) ou, no caso de impossibilidade, de seu familiar ou responsável. Antes da execução, o projeto de pesquisa foi avaliado pelo Comitê Permanente de Ética em Pesquisas com seres humanos (COPEP) da Universidade Estadual de Maringá, cuja aprovação está registrada no Parecer n. ${ }^{\circ}$ 132/2009, CAAE nº 0040-09.

\section{RESULTADOS E DISCUSSÃO}

\section{Qualidade dos Cuidados de Enfermagem}

O percentual das respostas positivas (Índice de Positividade) obtido para cada item que compôs o Roteiro de Auditoria Operacional, bem como a Classificação de Qualidade dos itens, está disposto na Tabela 1.

\section{Tabela 1 - Distribuição dos itens do Roteiro de Auditoria Operacional e respectivos Índices de Positividade e Classificação de Qualidade. Cascavel-PR, 2010.}

\begin{tabular}{l|c|c}
\hline $\begin{array}{c}\text { Itens do Roteiro de Auditoria } \\
\text { Operacional }\end{array}$ & $\begin{array}{c}\text { Índice de Posi- } \\
\text { tividade (\%) }\end{array}$ & $\begin{array}{c}\text { Classificação } \\
\text { de Qualidade }\end{array}$ \\
\hline I - Higiene e Conforto & 68 & Sofrível \\
II - Atividade Física & $\mathbf{2 8}$ & Sofrível \\
III - Segurança Física & 71 & Limítrofe \\
IV - Nutrição e Hidratação & 66 & Sofrível \\
V - Oxigenação e Ventilação & $\mathbf{2 9}$ & Sofrível \\
VI - Eliminações & 66 & Sofrível \\
VII - Utilização de Equipamentos & 72 & Limítrofe \\
VIII - Unidade de Terapia Intensiva & 50 & Sofrível \\
\hline MÉDIA & $\mathbf{5 6}$ & Sofrível \\
\hline
\end{tabular}

Classificação: Desejável: 100\% Adequada: 90-99\% Segura: 8089\% Limítrofe: 70-79\% Sofrível: < 70\%

Os cuidados de enfermagem executados na Unidade estudada foram avaliados por meio de oito itens. Destes, apenas os itens III - Segurança Física e VII - Utilização de Equipamentos obtiveram Índice de Positividade (IP) igual ou superior a 70\% e foram, portanto, considerados de qualidade (Tabela 1).

No item III - Segurança Física, apesar da avaliação conjunta dos subitens ter apresentado IP $\geq 70 \%$, ao serem analisados de forma individualizada, alguns obtiveram IP $\leq 70 \%$, como: "presença de campainha ao alcance do paciente" (IP=0\%); "limpeza do ambiente" - leito, mobiliário, ventilador, bombas infusoras, cabos de monitorização, entre outros - (IP=12\%); "presença de objetos limpos acumulados no boxe" (IP=40\%) e "presença de objetos com sujidades acumulados no boxe" (IP $=62 \%)$.

No tocante ao subitem "presença de campainha ao alcance do paciente", observou-se que esta forma de sinalização não ocorre em nenhum boxe. Este dado é preocupante porque demonstra a pouca importância atribuída ao sistema de sinalização em terapia intensiva. Acredita-se que este fato ocorre porque muitos pacientes, normalmente, se encontram em estado de inconsciência/coma e por isso não utilizam este recurso. Cabe lembrar, contudo, que mesmo em menor escala, existem pacientes internados na terapia intensiva que não estão em coma, e que necessitam utilizar algum dispositivo para solicitar a presença do profissional de enfermagem quando necessário e por este motivo, a campainha ou outro recurso do gênero deve estar disponível em todos os boxes da unidade.

Com relação ao tipo de recurso a ser disponibilizado para solicitar a presença da equipe da UTI, não precisa necessariamente que seja sonoro, o Ministério da Saúde (MS), recomenda que seja utilizado um sistema de sinalização, na forma de sinal luminoso, logo acima da cabeceira do leito do paciente, para que do posto de enfermagem seja possível à enfermagem, observar todos os boxes visualmente ou de forma eletrônica ${ }^{(11)}$.

Em relação ao subitem "limpeza do ambiente", que não alcançou o critério de qualidade proposto para este estudo (IP $\geq 70 \%$ ), observou-se, por diversas vezes, a presença de sujidade na bancada onde as bombas infusoras eram posicionadas; no monitor multiparamétrico; nos cabos de monitorização e no ventilador mecânico, demonstrando que a limpeza concorrente não estava sendo realizada a contento.

A limpeza concorrente é uma atividade da equipe de enfermagem que deve ser realizada diariamente, com água e sabão em todo mobiliário e demais equipamentos presentes no quarto/boxe do paciente ${ }^{(12)}$. Esta prática é indispensável em ambiente de terapia intensiva, porque este é considerado como ambiente crítico, onde os pacientes têm maior risco de adquirir infecção, devido ao fragilizado estado imunológico em que se encontram, associado ao elevado número de procedimentos invasivos a que são submetidos.

Outros dois subitens que também apresentaram IP $<70 \%$ se relacionam à "presença de objetos limpos acumulados no boxe" e à "presença de objetos com sujidades acumulados no boxe". Dentre os objetos limpos observados em excesso, destacam-se os de uso coletivo, como tubos de pomadas; rolos de esparadrapo; dentre outros, que deveriam permanecer no Posto de Enfermagem. Além dos materiais de uso coletivo, foi observado também o excesso de materiais nas caixas de apoio presentes nos boxes, como luvas e sondas de aspiração, pacotes de gaze, agulhas, seringas, fitas para verificação de glicemia, os quais deveriam estar presentes somente na quantidade necessária para o uso em cada plantão.

No que concerne aos materiais com sujidades observados nos boxes, os mais frequentes foram os que se referiam aos curativos de punção venosa, além de comadres e papagaios. Sabe-se que estes objetos abrigam grande quantidade de materiais contaminantes e, por isso, a não destinação adequada dos mesmos implica em riscos à segurança do paciente e do profissional, podendo ocasionar infecções e/ou acidentes ocupacionais.

$\mathrm{Na}$ terapia intensiva, a ocorrência de acidentes ocupacionais com profissionais de enfermagem decorre, principalmente, da maior demanda de procedimentos invasivos presentes na unidade, do intenso ritmo de trabalho da equipe e do quadro reduzido de pessoal ${ }^{(13)}$. Esses fatores, juntamente com a 
presença de sujidades e o excesso de materiais nos boxes devem ser minimizados porque, sem dúvida, são sinônimos de riscos e/ou prejuízos à equipe, ao cliente e à instituição.

Apesar de alguns subitens referentes à segurança do paciente terem obtido IP $<70 \%$, no âmbito geral, este item foi considerado de qualidade. Exemplo disso é o item VII - Utilização de Equipamentos, composto por apenas um subitem, relacionado à utilização da forma correta dos equipamentos como oxímetro de pulso, transdutores de pressão (venosa, abdominal e cerebral), cardioscópios, dentre outros que são utilizados na monitorização dos pacientes internados em terapia intensiva.

A monitorização na terapia intensiva, segundo o Consenso Brasileiro de Monitorização e Suporte Hemodinâmico ${ }^{(14)}$, é considerada como uma das mais importantes ferramentas no tratamento dos pacientes críticos. Desse modo, deve ser realizada de forma correta, principalmente pela equipe de enfermagem, porque os profissionais que a compõe são os que manuseiam monitores com maior frequência.

Neste estudo os cuidados concernentes à utilização de equipamentos foram considerados de qualidade, no entanto o IP obtido situou-se no limite inferior (72\%) e foi classificado como "Limítrofe", demonstrando que são necessárias medidas que visem o aperfeiçoamento da equipe no manuseio dos equipamentos utilizados na unidade.

Os demais itens avaliados pela Auditoria Operacional apresentaram IP $<70 \%$. Dessa forma, alcançaram valores que se situaram no nível de classificação "Sofrível".

Dentre os itens classificados como "Sofríveis", tem-se o de número I - Higiene e Conforto $(\mathrm{IP}=68 \%)$ que apresentou IP $<70 \%$ nos seguintes subitens: "aparamento das unhas dos pés e mãos" (26\%); "realização de tricotomia facial" (54\%) e "correto posicionamento do paciente na cama" (64\%).

Os três subitens referidos são atividades realizadas para melhorar as condições de aparência, higiene e conforto, tidas como técnicas básicas de enfermagem. Na UTI-A, os cuidados referentes a essas necessidades não alcançaram o critério de qualidade, o que é preocupante, pois consistem em técnicas que não requerem profundo conhecimento científico, nem muita habilidade para serem realizadas. Com base nestes dados, conjectura-se que os profissionais de enfermagem da Unidade não valorizam os cuidados denominados de técnicas básicas.

Outro item que não obteve IP de acordo com o critério de qualidade foi o de número IV - Nutrição e Hidratação $(\mathrm{IP}=66 \%)$, o qual apresentou IP $<70 \%$ nos seguintes subitens: "troca dos equipos de microgotas a cada 72 horas" (IP = 54\%); "identificação correta dos equipos de soro/medicações" (IP = 34\%); "identificação completa e correta do soro/ medicações instalados" (IP =0\%); "identificação das punções venosas periféricas realizadas" (IP $=0 \%)$ e "troca das punções venosas periféricas a cada 72 horas" (IP $=50 \%)$.

A troca dos equipos deve ser realizada a cada 72 horas, conforme determina a Comissão de Controle de Infecção Hospitalar $(\mathrm{CClH})$ da instituição investigada, que se pauta nas diretrizes para prevenção de infecções relacionadas a cateteres intravasculares do Center for Disease Control and
Prevention (CDC) ${ }^{(15)}$, contudo foi observado que, na UTI-A, esta recomendação não está sendo cumprida.

A importância da troca frequente dos equipos se deve ao fato de que este artefato se liga diretamente ao acesso venoso e, quando ocorre necessidade de desconexão entre o equipo e o frasco de soro/medicação, pode ocorrer o contato inadvertido da ponta do equipo com as mãos do profissional, ou qualquer outro objeto que esteja próximo, gerando contaminação e exposição do sistema estéril(16).

No que tange à "identificação correta dos equipos de soro/ medicações", foi observado que, apesar de existirem etiquetas padronizadas para tal prática, muitas vezes o preenchimento foi realizado de forma incorreta, ou não realizado. Falhas desse tipo podem ter contribuído para que a troca dos equipos não fosse realizada de acordo com o que estabelece a $\mathrm{CCIH}$ do Hospital e o CDC ${ }^{(15)}$.

Em relação ao subitem referente à "identificação correta e completa dos soros/medicações instalados", os principais itens observados foram: o registro de forma incorreta ou incompleta do nome do paciente, do tempo e da vazão de administração da solução.

Quanto ao nome, foi observada a falta de registro do sobrenome, podendo comprometer a segurança dos pacientes, em razão de que pode gerar dúvidas e erros no momento da instalação da solução, principalmente se existirem pacientes com nome semelhante ou igual na unidade.

Com relação ao registro incompleto, incorreto ou mesmo não registro do tempo e da vazão em que a solução deve ser administrada no rótulo do soro/medicações, isso se deve, em parte, ao fato de que, na UTI, é constante a utilização das bombas infusoras, as quais, após o informe do volume e do tempo em que a solução deve ser infundida, automaticamente calculam a vazão por hora, não sendo necessário o cálculo anterior pela equipe de enfermagem. Isso resulta na ausência do registro prévio, também, da vazão e do tempo, porque o cálculo é realizado pela própria bomba, após a instalação da solução, momento em que, normalmente, o rótulo já foi preenchido e aderido ao frasco. Apesar dessas considerações, a não necessidade do cálculo manual da vazão de infusão da solução não exime a equipe de enfermagem de completar o rótulo, posteriormente à programação da bomba infusora.

No subitem relacionado à "identificação correta das punções venosas periféricas" foi observado que, normalmente, faltava algum dos dados requeridos como: nome do profissional que realizou a punção e data e/ou horário da realização do procedimento. A identificação incompleta ou ausente das punções venosas promove, muitas vezes, a necessidade de realização de um novo procedimento e isso, certamente, é prejudicial, principalmente para o paciente, por elevar o risco de contrair infecção e provocar dor e desconforto no mesmo.

Outro item que também não obteve IP de acordo com o critério de qualidade estabelecido foi o de número VI - Eliminações $(I P=66 \%)$. Para este item os subitens que apresentaram IP $<70 \%$ foram: "instalação adequada do dreno de tórax" $(\mathrm{IP}=67 \%)$; "correto posicionamento da sonda nasogástrica e nasoenteral" (IP $=55 \%)$ e "adequada fixação de cateter vesical de demora" $(\mathrm{IP}=9 \%)$. 
No subitem relacionado à "instalação adequada do dreno de tórax" foram avaliados os cuidados considerados de menor complexidade, tanto no aspecto técnico, quanto no científico como é o caso da realização de curativos diários ao redor do dreno; manutenção do frasco coletor abaixo do nível do tórax do paciente e, mesmo assim, este cuidado foi considerado "Sofrível".

A classificação baixa dos cuidados referentes à "instalação adequada do dreno de tórax" preocupa, visto que os cuidados avaliados são simples de serem realizados em relação a outros requeridos por pacientes em uso de dreno de tórax, como: monitoração de sinais e sintomas de pneumotórax; monitoramento de enchimento, esvaziamento/eliminação e vazamento de ar por meio do dreno torácico ${ }^{(17)}$, os quais não foram avaliados neste estudo.

No subitem pertinente ao "correto posicionamento da sonda nasogástrica/nasoenteral (SNG/SNE)", observou-se, nos pacientes em ventilação mecânica, que a fixação foi realizada juntamente com o tubo endotraqueal, de modo que, quando havia qualquer movimento do tubo, a sonda também era movimentada, facilitando a retirada da mesma. Outra forma de fixação da SNG/SNE observada foi na região superior do nariz, causando pressão na região da asa do nariz e podendo causar úlcera por pressão (UP).

A não fixação da SNG/SNE de forma correta, que, de acordo com a literatura, deve ser realizada sem tracionar a asa do nariz, com uma fita antialérgica, a qual deve ser trocada sempre que estiver úmida, frouxa, ou solta ${ }^{(18)}$, pode facilitar que este dispositivo seja retirado pelo paciente, ou tracionado pela equipe de enfermagem no momento da execução dos cuidados. Acidente desse tipo é um grande inconveniente, porque a retirada de sondas consiste em um evento adverso que pode ser utilizado como indicador de resultado da qualidade dos cuidados de enfermagem ${ }^{(19)}$.

Na UTI-A não existe registro sobre as retiradas não programadas de SNG/SNE. Entretanto, considerando que os cuidados de enfermagem relacionados à correta fixação da sonda não obtiveram IP de acordo com o critério de qualidade determinado, percebe-se que há necessidade de se realizar estudos com objetivo de verificar o número e as ocasiões em que as sondas são retiradas sem indicação, para que assim se fundamentem treinamentos da equipe de enfermagem sobre a importância de maior atenção no cuidado e na manipulação deste artefato.

Com relação ao subitem "adequada fixação do cateter vesical de demora (CVD)", e "adequada fixação da Sonda Nasogástrica (SNG) e Nasoenteral (SNE)", observaram-se falhas na sua realização, principalmente no prolongamento do CVD que, costumeiramente, não era fixado, mantendo-se o tempo todo tracionado. Apesar desse inconveniente, a maior problemática na questão da inadequada ou ausente fixação do CVD, não consiste na retirada não programada, mas sim na lesão que pode causar nas estruturas da bexiga e da uretra, devido à constante movimentação da mesma ${ }^{(20)}$.

O item VIII - Unidade de Terapia Intensiva, não obteve $I P \geq 70 \%$, portanto, não atingiu o critério de qualidade, devido à presença de subitens com IP $<70 \%$, quais sejam: "presença de prescrição de enfermagem para todos os pacientes" $(\mathrm{IP}=36 \%)$; "presença mínima de duas evoluções diárias"
$(\mathrm{IP}=28 \%)$; "registro da realização de um exame físico por período" (IP =4\%); "presença de cuidados preventivos para UP" $(\mathrm{IP}=32 \%)$; "presença de controle de ruídos no setor" (IP $=0 \%)$ e "realização de antissepsia nas mãos, antes e após procedimentos no setor" (IP=15\%).

$\mathrm{O} I \mathrm{P}<70 \%$ para os subitens referentes à "realização de prescrição de enfermagem diariamente para todos os pacientes", "presença mínima de duas evoluções diárias" e "registro da realização de um exame físico" são etapas referentes à Sistematização da Assistência de Enfermagem (SAE), metodologia que ainda não está implantada de forma sistemática na Instituição e também na UTI-A. Verificou-se que, na UTI-A, normalmente, as prescrições de enfermagem e as evoluções, quando presentes no prontuário dos pacientes, eram realizadas pelos acadêmicos de enfermagem, em estágio curricular, sob supervisão do enfermeiro da Unidade. Quanto ao exame físico, este não era realizado de maneira frequente e quando executado, anotava-se na própria evolução, somente alguns dados referentes às principais anormalidades observadas no paciente.

A realização da SAE consiste em uma atividade privativa do profissional enfermeiro e é entendida como uma prática que promove avanços na qualidade do cuidado de enfermagem, visto que possibilita o planejamento e o desenvolvimento de ações fundamentadas cientificamente e de acordo com as necessidades de cada paciente ${ }^{(21)}$.

Em relação ao subitem pertinente à "presença de cuidados preventivos para UP", foi observado que a mudança de decúbito, normalmente recomendada a cada duas horas ${ }^{(22-23)}$, não era realizada na frequência prescrita. A utilização de colchões de ar e coxins para aliviar a pressão nas regiões propensas à formação de UP também não era realizada adequadamente, em razão da ausência, ou mesmo da insuficiência desses equipamentos.

A questão da insuficiência, da ausência e mesmo da inadequação dos materiais utilizados para a prevenção de UP na Unidade, requer ações no âmbito gerencial, no sentido de pleitear a ampliação do número ou mesmo a inclusão de novos itens na lista de materiais médico-hospitalares a serem adquiridos pela Instituição, para visar solucionar esta problemática.

O subitem "presença de controle de ruídos na unidade", não atingiu o critério de qualidade, pois obteve $\mathrm{IP}=0 \%$. Cabe destacar que a observação foi realizada nos primeiros $30 \mathrm{mi}-$ nutos de cada plantão, período que corresponde à passagem de plantão entre os profissionais que atuam na Unidade e por isso apresenta maior agitação e ruídos. Salienta-se que não foi utilizado um analisador de ruídos, aparelho considerado ideal para mensurar precisamente o nível de ruído dos ambientes, de modo que a observação, neste estudo, baseou-se na percepção empírica da pesquisadora.

O excesso de ruídos, especialmente na terapia intensiva, ocorre porque essas unidades possuem grande diversidade de equipamentos com alarmes acústicos, além da conversa frequente da equipe. $\mathrm{O}$ exagero de ruídos torna o ambiente de UTI estressante, podendo causar distúrbios fisiológicos (no sono e no ritmo cardíaco) e psicológicos (desorientação, ansiedade, irritabilidade e psicose de UTI) tanto nos pacientes, como na equipe que atua na unidade ${ }^{(24)}$, o que pode prejudicar as atividades laborais e comprometer a qualidade do cuidado. 
A "realização de antissepsia das mãos antes e após procedimentos no setor" (IP $=15 \%)$, subitem que também não alcançou o critério de qualidade, consiste em uma prática primordial não somente na terapia intensiva, mas em todos os setores do hospital. Contudo, na UTI, esta prática se reveste de vital importância devido à intensa realização de procedimentos invasivos nos pacientes que, normalmente, se encontram mais susceptíveis às infecções ${ }^{(25)}$.

Cabe salientar que a avaliação sobre a lavagem das mãos, na Unidade, apesar de ter sido realizada em curto espaço de tempo (30 minutos), permitiu observar que esta prática não foi executada de maneira sistemática, antes e após o contato com os pacientes. Esse dado confirma a importância da atualização/capacitação da equipe visando maior conscientização acerca da realização deste procedimento que é simples, necessário e muito eficaz.

Dentre os seis itens que apresentaram IP $<70 \%$ e não alcançaram o critério de qualidade, destacam-se dois, por terem obtido IP extremamente baixos: Itens II - Atividade Física $(\mathrm{IP}=28 \%)$ e V - Oxigenação e Ventilação (IP $=29 \%)$. Dados como estes certamente geram apreensão e insegurança nos trabalhadores, porque significam riscos e prejuízos à clientela.

Com relação ao item II - Atividade Física, todos os seus subitens tiveram IP $<70 \%$. Destaca-se que os subitens que o compuseram se relacionam com a imobilidade do paciente e com medidas que visam minimizar a impossibilidade de movimentação. A importância da avaliação deste item se deve aos prejuízos que podem ser atribuídos à imobilidade física, seja ela parcial ou total, como a ocorrência de atrofia muscular, anasarca e, principalmente, o desenvolvimento de UP.

Na Unidade estudada, já no terceiro dia foram observadas UPs e mesmo escara, que consiste em presença de áreas necróticas, principalmente na orelha externa, próximo à região da hélix, local onde se posiciona o cadarço utilizado para fixar o tubo endotraqueal do paciente em ventilação mecânica. O referido local onde foi observada a UP nos pacientes auditados não coaduna com os dados descritos na literatura, que apresenta como locais mais freqüentes, as regiões sacral, calcânea e escapular ${ }^{(22-23)}$.

Conforme foi dito anteriormente, os cuidados de enfermagem com a fixação do tubo endotraqueal ou mesmo o material utilizado para a sua fixação, parecem estar inadequados. Para se certificar disso com mais precisão, é preciso que novas possibilidades de fixação sejam buscadas e que sejam realizadas capacitações da equipe de enfermagem, a fim de que as UP na região da orelha, observadas neste estudo, possam ser evitadas.

$\mathrm{O}$ segundo item que apresentou IP extremamente abaixo do critério de qualidade estabelecido para este estudo, sendo classificado como "Sofrível", foi o item V - Oxigenação e Ventilação $(I P=29)$, para o qual todos os subitens também apresentaram IP $<70 \%$. Os referidos subitens se relacionam a aspectos técnicos básicos dos cuidados de enfermagem que são necessários aos pacientes em uso de $\mathrm{O}_{2}$ suplementar, seja pelo modo espontâneo ou artificial. Apesar da baixa complexidade na realização dos cuidados auditados, estes não foram classificados como sendo de qualidade, o que aponta que é preciso efetuar ações de melhorias junto à equipe, também no âmbito da oxigenoterapia.

A necessidade de melhorar os cuidados referentes à oxigenação e ventilação se deve ao fato que os cuidados, com qualidade prejudicada, podem causar sérios danos aos pacientes, como a piora das condições clínicas e a extubação acidental ${ }^{(26-27)}$.

$\mathrm{Na}$ UTI-A deste estudo não existe notificação das consequências e mesmo dos danos causados aos pacientes no âmbito da oxigenação e ventilação. Com isso, considera-se importante a implantação da prática da notificação das intercorrências com relação a esses procedimentos, uma vez que os cuidados prestados neste serviço, não alcançaram o critério de qualidade estabelecido.

De acordo com os dados deste estudo, de maneira geral, os cuidados prestados na UTI-A, avaliados por meio dos oito itens do Roteiro de Auditoria Operacional, apresentaram IP médio de $56 \%$ e, assim, a maioria dos itens e respectivos subitens não atingiram o critério de qualidade proposto.

\section{CONSIDERAÇÕES FINAIS}

Com base nos resultados apresentados a qualidade dos cuidados de enfermagem prestados na UTI-A estudada foi observada somente nos itens III - Segurança Física (IP =71\%) e VII - Utilização de Equipamentos (IP = 72\%), por terem alcançado IP $\geq 70 \%$. Entretanto o IP destes itens foi considerado apenas como "Limítrofe", de modo que existe espaço para melhorias nos cuidados relacionados aos mesmos.

Os cuidados de enfermagem dos demais itens avaliados, não foram considerados de qualidade uma vez que apresentaram IP $<70 \%$. Há que se destacar os itens II - Atividade Física (28\%) e V - Oxigenação e Ventilação (29\%) por terem apresentado os menores IP do estudo.

A avaliação dos cuidados de enfermagem da UTI-A por meio da Auditoria Operacional permitiu conhecer a qualidade de parte dos cuidados desenvolvidos neste serviço e os seus dados, não só contribuem ao direcionamento de ações de meIhoria à equipe investigada, como também a outros Serviços.

\section{REFERÊNCIAS}

1. Moraes AS, Braga AT, Nicole AG, Tronchin DMR, Melleiro MM. Qualidade e avaliação em saúde: publicações em periódicos de enfermagem nas últimas duas décadas. Rev. enferm. UERJ. 2008; 16(3): 404-9.
2. Matsuda LM, Évora YDM, Boan FS. O método desdobramento da função qualidade - QFD - no planejamento do serviço de enfermagem. Rev. Latino-Am. Enfermagem. 2000; 8(5): 97-105. 
3. D'Innocenzo M, Adami NP, Cunha ICKO. O movimento pela qualidade nos serviços de saúde e enfermagem. Rev Bras Enferm. 2006; 59(1): 84-8.

4. Matsuda LM. Satisfação profissional da equipe de enfermagem na UTI-adulto: perspectivas de gestão para a qualidade da assistência. [Tese]. Ribeirão Preto (SP): Escola de Enfermagem de Ribeirão Preto, Universidade de São Paulo; 2002. 262 f. Doutorado em Enfermagem.

5. Rodrigues VA, Perroca MG, Jericó MC. Glosas hospitalares: importância das anotações de enfermagem. Arq Ciênc Saúde. 2004; 11(4): 210-14.

6. Kurcgant P. Administração em enfermagem. São Paulo: EPU; 2006.

7. Haddad MCL. Qualidade da assistência de enfermagem: processo de avaliação de um hospital universitário público. [Tese]. Ribeirão Preto (SP): Escola de Enfermagem de Ribeirão Preto, Universidade de São Paulo; 2004. 201 f. Doutorado em Enfermagem.

8. D’Innocenzo M. Auditorias em serviços de saúde e enfermagem: uma introdução. In: D'linnocenzo M. (Coord.). Indicadores, auditorias, certificações: ferramentas de qualidade para gestão em saúde. São Paulo: Martinari, 2006. cap. 7, p. 121-127.

9. Setz VG, D'Innocenzo M. Avaliação da qualidade dos registros de enfermagem por meio da auditoria. Acta paul. enferm. 2009; 22(3): 313-7.

10. Saupe H, Horr L. Auditoria em enfermagem. Revista Ciên Saúde. 1982; 1(1): 23.

11. Brasil. RDC n. 50 de 21 de fevereiro de 2002. Regulamento Técnico para Planejamento, Programação, Elaboração e Avaliação de Projetos Físicos de EAS. Agência Nacional de Vigilância Sanitária. Brasília; 2002. [acesso 05 dez 2009]. Disponível em: < http://www.anvisa.gov. br/anvisalegis/resol/2002/50_02rdc.pdf > .

12. Zorze CMFS, Karagulian PP. In: Murta GF. Saberes e práticas: guia para ensino e aprendizado de enfermagem. 2ed. São Caetano do Sul: Difusão Editora; 2006. p. 101-18.

13. Bonini AM, Zevian CP, Facchin LT, Gir E, Canini SRMS. Exposição ocupacional dos profissionais de enfermagem de uma unidade de terapia intensiva a material biológico. Rev. Eletr. Enf. [Internet]. 2009; 11(3): 658-64.

14. Dias FS, Resende E, Mendes CL, Néto AR, David CM, Schettino G, Lobo SMAAB, et al. Parte II: monitorização hemodinâmica básica e cateter de artéria pulmonar. Rev. bras. ter. intensiva. 2006; 1(1): 63-77.

15. O'Grady NP, Alexander M, Dellinger EP, Gerberding JL, Heard SO, Maki DG, Masur H, McCormick RD, Mermel LA, Pearson ML, Raad II, Randolph A, Weinstein RA.
Guidelines for the prevention of intravascular catheterrelated infections. MMWR Morb Mortal Wkly Rep. 2002; 23(12): 759-69.

16. Martins KA, Tipple AFV, Souza ACS, Barreto RASS, Siqueira KM, Barbosa JM. Adesão às medidas de prevenção e controle de infecção de acesso vascular periférico pelos profissionais da equipe de enfermagem. Cienc Cuid Saúde. $2008 ; 7(4)$ : 485-92.

17. Dochterman JM, Bulechek GM. Classificação das intervenções de enfermagem (NIC). 4ed. Porto Alegre: Artmed; 2008.

18. Poltronieri MJA. Terapia nutricional enteral. In: PadiIha KG, Vatimo MFF, Silva SC, Kimura M. Enfermagem em UTI: cuidando do paciente crítico. Barueri: Manole; 2010. p. 672-97.

19. Nascimento KC, Backes DS, Koerich MS, Erdmann AL. Sistematização da assistência de enfermagem: vislumbrando um cuidado interativo, complementar e multiprofissional. Rev Esc Enferm USP. 2008; 42(4): 643-648.

20. Napoleão AA, Caldato VG, Petrilli Filho JF. Diagnósticos de enfermagem para o planejamento da alta de homens prostatectomizados: um estudo preliminar. Rev. Eletr. Enf. [Internet]. 2009; 11(2): 286-94.

21. Trupell TC, Meier MJ, Calixto RC, Peruzzo SA, Crozeta K. Sistematização da assistência de enfermagem em unidade de terapia intensiva. Rev Bras Enferm. 2009; 62(2): 221-7.

22. Fernandes LM, Caliri MHL. Uso da escala de Bradem e Glasgow para identificação do risco para úlcera de pressão em pacientes internados em centro de terapia intensiva. Rev. Latino-Am. Enfermagem. 2008; 6(16): 973-8.

23. Rocha $A B L$, Barros $S M O$. Avaliação e risco de úlcera de pressão: propriedades de medida da versão em português a escala de Waterlow. Acta paul. enferm. 2009; 2(20): 143-150.

24. Pereira RP, Toledo RN, Amaral JLG, Guilherme A. Qualificação e quantificação a exposição sonora ambiental em uma unidade de terapia intensiva geral. Rev Bras Otorrinolaringol. 2003; 69(6): 766-71.

25. Mendonça M. Serviço de controle de infecções hospitalares na prevenção da pneumonia associada à ventilação mecânica. Prática Hospitalar. 2009; 11(66): 55-56.

26. Barbosa AL, Campos ACS, Chaves EMC. Complicações não clínicas da ventilação mecânica: ênfase no cuidado de enfermagem neonatal. Acta paul. enferm. 2006; 19(4): 439-43.

27. Castellões TMFW, Silva LD. Ações de enfermagem para a prevenção da extubação acidental. Rev Bras Enferm. 2009; 62(4): 540-5. 